\title{
Hemodynamic Differences in Intracranial Aneurysms before and after Rupture
}

B.M.W. Cornelissen, J.J. Schneiders, W.V. Potters, R. van den Berg, B.K. Velthuis, G.J.E. Rinkel, C.H. Slump, E. VanBavel, C.B.L.M. Majoie, and H.A. Marquering

\begin{abstract}
BACKGROUND AND PURPOSE: Rupture risk of intracranial aneurysms may depend on hemodynamic characteristics. This has been assessed by comparing hemodynamic data of ruptured and unruptured aneurysms. However, aneurysm geometry may change before, during, or just after rupture; this difference causes potential changes in hemodynamics. We assessed changes in hemodynamics in a series of intracranial aneurysms, by using 3D imaging before and after rupture.
\end{abstract}

MATERIALS AND METHODS: For 9 aneurysms in 9 patients, we used MRA, CTA, and 3D rotational angiography before and after rupture to generate geometric models of the aneurysm and perianeurysmal vasculature. Intra-aneurysmal hemodynamics were simulated by using computational fluid dynamics. Two neuroradiologists qualitatively assessed flow complexity, flow stability, inflow concentration, and flow impingement in consensus, by using flow-velocity streamlines and wall shear stress distributions.

RESULTS: Hemodynamics changed in 6 of the 9 aneurysms. The median time between imaging before and after rupture was 678 days (range, 14-1461 days) in these 6 cases, compared with 151 days (range, 34-183 days) in the 3 cases with unaltered hemodynamics. Changes were observed for flow complexity $(n=3)$, flow stability $(n=3)$, inflow concentration $(n=2)$, and region of flow impingement $(n=3)$. These changes were in all instances associated with aneurysm displacement due to rupture-related hematomas, growth, or newly formed lobulations.

CONCLUSIONS: Hemodynamic characteristics of intracranial aneurysms can be altered by geometric changes before, during, or just after rupture. Associations of hemodynamic characteristics with aneurysm rupture obtained from case-control studies comparing ruptured with unruptured aneurysms should therefore be interpreted with caution.

ABBREVIATIONS: CFD = computational fluid dynamics; 3DRA = 3D rotational angiography; OSI = oscillatory shear index; WSS = wall shear stress

ntracranial aneurysms are found in $1 \%-5 \%$ of the adult population. ${ }^{1,2}$ For ruptured intracranial aneurysms, case morbidity and fatality rates are high. ${ }^{1,3}$ However, $50 \%-80 \%$ of all intracra-

Received October 24, 2014; accepted after revision March 3, 2015.

From the MIRA Institute for Biomedical Engineering and Technical Medicine (B.M.W.C., C.H.S.), University of Twente, Enschede, the Netherlands; Departments of Radiology (B.M.W.C., J.J.S., W.V.P., R.v.d.B., C.B.L.M.M., H.A.M.) and Biomedical Engineering and Physics (B.M.W.C, E.V., H.A.M.), Academic Medical Center, Amster dam, the Netherlands; and Departments of Radiology (B.K.V.) and Neurology and Neurosurgery, Brain Center Rudolf Magnus (G.J.E.R.), University Medical Center Utrecht, Utrecht, the Netherlands.

C.B.L.M. Majoie and H.A. Marquering contributed equally to this work.

This work was supported by a grant from the NUTS-OHRA Foundation (grant no. SNO-T-0702-92) and a grant from the Dutch Technology Foundation STW (CARISMA 11629).

Please address correspondence to B.M.W. Cornelissen, MSc, Meibergdreef 9, 1105 AZ, Amsterdam, the Netherlands; e-mail: b.m.cornelissen@amc.uva.nl

-- Indicates open access to non-subscribers at www.ajnr.org

= Indicates article with supplemental on-line table.

DIndicates article with supplemental on-line video.

http://dx.doi.org/10.3174/ajnr.A4385 nial aneurysms do not rupture during an individual's lifetime. ${ }^{1}$ More commonly, unruptured aneurysms are incidentally found due to increasing use of imaging. ${ }^{4,5}$ The risk of rupture should be balanced against the risk of treatment when deciding whether an aneurysm should be treated. In clinical practice, the location and size of the aneurysm are the most important parameters for determining the risk of rupture. ${ }^{1,6}$ However, these geometric predictors are insufficient for optimal treatment selection. Therefore, the search for better predictors for rupture continues. ${ }^{7-9}$ Previous studies have associated intra-aneurysmal flow patterns and wall shear stress (WSS) distributions with aneurysm rupture status. ${ }^{7,8,10}$ However, these results are still controversial. For example, both high and low aneurysmal WSS were separately associated with aneurysm growth and rupture. ${ }^{11,12}$ In these risk-assessment studies, potential changes in hemodynamics due to the rupture itself were systematically neglected. Recently, 2 studies have shown changes in aneurysm geometry after rupture. ${ }^{13,14}$ These rupture-associated geometric changes may result in differences in hemodynamic characteristics as well. 
Table 1: Imaging modalities for all 9 patients before and after rupture, with the time between imaging before and after rupture and the days between hemorrhage and imaging after rupture ${ }^{a}$

\begin{tabular}{|c|c|c|c|c|c|c|}
\hline $\mathbf{P t}$ & $\begin{array}{l}\text { Imaging Modality } \\
\text { before Rupture }\end{array}$ & Resolution (mm) & $\begin{array}{c}\text { Imaging Modality } \\
\text { after Rupture }\end{array}$ & Resolution (mm) & $\begin{array}{l}\text { Time between } \\
\text { Imaging before and } \\
\text { after Rupture }\end{array}$ & $\begin{array}{c}\text { Days between } \\
\text { Hemorrhage and } \\
\text { Imaging after Rupture }\end{array}$ \\
\hline 1 & 3D TOF MRA & $0.49 \times 0.49 \times 1.2$ & 3DRA & $0.22 \times 0.22 \times 0.22$ & 0 yr, 183 days & 1 \\
\hline 2 & 3D TOF MRA & $0.31 \times 0.31 \times 1.0$ & CTA & $0.31 \times 0.31 \times 0.45$ & 0 yr, 72 days & 1 \\
\hline 3 & CTA & $0.33 \times 0.33 \times 1.0$ & CTA & $0.35 \times 0.35 \times 0.33$ & 2 yr, 323 days & 2 \\
\hline 4 & 3D PC MRA & $0.78 \times 0.78 \times 1.0$ & 3DRA & $0.25 \times 0.25 \times 0.25$ & $0 \mathrm{yr}, 151$ days & 0 \\
\hline 5 & 3D TOF MRA & $0.45 \times 0.45 \times 1.0$ & 3DRA & $0.39 \times 0.39 \times 0.39$ & 3 yr, 148 days & 0 \\
\hline 6 & CTA & $0.43 \times 0.43 \times 0.9$ & 3DRA & $0.22 \times 0.22 \times 0.22$ & 0 yr, 34 days & 0 \\
\hline 7 & 3D TOF MRA & $0.20 \times 0.20 \times 1.0$ & 3DRA & $0.17 \times 0.17 \times 0.17$ & $4 \mathrm{yr}, 1$ day & 3 \\
\hline 8 & 3D TOF MRA & $0.35 \times 0.35 \times 1.4$ & 3DRA & $0.17 \times 0.17 \times 0.17$ & 0 yr, 14 days & 9 \\
\hline 9 & CTA & $0.33 \times 0.33 \times 1.3$ & 3DRA & $0.09 \times 0.09 \times 0.09$ & $0 \mathrm{yr}, 302$ days & 2 \\
\hline
\end{tabular}

Note:-Pt indicates patient; PC, phase-contrast.

a Table adapted from Schneiders et al. ${ }^{14}$

In this study, we had the opportunity to use high-quality 3D imaging data of 9 patients with intracranial aneurysms, obtained before and after rupture, to assess potential differences in hemodynamic characteristics associated with rupture.

\section{MATERIALS AND METHODS}

3D image data of intracranial aneurysms for 9 patients before (MRA $[n=6]$, CTA $[n=3]$ ) and after (CTA $[n=2]$, 3D rotational angiography [3DRA, $n=7]$ ) rupture were collected. The imaging parameters are shown in Table $1 .{ }^{14}$ Clinical data and aneurysmal geometric data are shown in the On-line Table. The image data were collected during 6 years in 3 different institutions. Four male and 5 female patients were included, with aneurysms located at the basilar artery $(n=3)$, posterior communicating artery $(n=2)$, middle cerebral artery $(n=2)$, anterior communicating artery $(n=1)$, and anterior choroidal artery $(n=$ 1). The mean age at the time of rupture was $60 \pm 12$ years. The median time between imaging before and after rupture was 183 days, with a range of 14 days to 4 years 1 day. The median time between rupture and subsequent imaging was 1 day, with a range of 0-9 days. Informed consent was waived by the medical ethics committee because no diagnostic tests other than routine clinical imaging were used in this retrospective study.

\section{Vascular Model Generation}

To create a surface model of the aneurysm and perianeurysmal vasculature by using the available $3 \mathrm{D}$ imaging data, we used a level set algorithm of the Vascular Modeling Toolkit (http://www .vmtk.org/). ${ }^{15-18}$ For hemodynamic simulation, model accuracy and mesh quality are important. ${ }^{18-20}$ Therefore, inaccuracies due to imaging artifacts such as flow dispersion and saturation (in 3D time-of-flight MRA), partial voluming, beam hardening (in CTA), neck size overestimation, ${ }^{21}$ and inhomogeneous contrast agent distribution (in CTA and 3DRA) were corrected. Artificial narrowing results in overestimations of WSS and erroneous flow velocities $^{22}$ and was, therefore, corrected by inflation by using Blender (http://www.blender.org). ${ }^{23}$ Vessel inflation was performed in the proximal arteries in 13 models and in the distal arteries in 12 models. Furthermore, the inflow jet and location of deflection may be sensitive to the segmentation of the neck area. To reduce this dependency, an experienced neuroradiologist (C.B.L.M.M.) carefully inspected the vascular models and corrected them for imaging artifacts. Imaging artifacts were distin- guished from pathology by using additional DSA imaging. For the purpose of this study, manual corrections of the segmentation were performed by using ITK-SNAP 2.4.0 (www.itksnap.org). ${ }^{24}$ In addition, the surface models were smoothed by using a built-in Taubin-smoothing algorithm of the Vascular Modeling Toolkit to remove high-frequency noise while preventing the surface models from shrinking. ${ }^{25}$

Subsequently, all aneurysm models were inspected for apparent differences in visualized anatomy of perianeurysmal arteries before and after rupture. The vascular models agreed for all except 1 case. In case 8, we removed 2 outflow arteries from the ruptured model because in the before-rupture imaging, only 1 outflow artery was visible, while 3 outflow arteries were visible in the afterrupture imaging.

To decrease computational effort, we removed distant vessel branches located approximately 20 times the average radius distal to the aneurysm. Finally, the in- and outlet vessels were cut perpendicular to the vessel. To ensure fully developed flow entering the aneurysm model, we added cylindrical flow extensions, with a length of 12 times the radius of the afferent vessel, to the inlets of the models. ${ }^{18,26}$

All surface models were converted into tetrahedral meshes by using the Vascular Modeling Toolkit. ${ }^{18}$ The element attenuation close to the wall was increased by adding a customized boundary layer. The median number of elements was 3,284,000 (range $2,101,000-5,248,000)$.

\section{Computational Fluid Dynamics}

Computational fluid dynamics (CFD) (Fluent 13.0; ANSYS, Canonsburg, Pennsylvania) was used to simulate hemodynamics in the vascular models. Transient Navier-Stokes equations were solved by using a pressure-based, 3D double-precision solver by following the SIMPLE Method. ${ }^{27}$ Blood was modeled with an attenuation of $1060 \mathrm{~kg} / \mathrm{m}^{3}$ and a dynamic viscosity of $0.004 \mathrm{~Pa} \cdot \mathrm{s}$. A no-slip boundary was assumed at the rigid vessel wall.

Because patient-specific velocity data were not available for this patient selection, we calculated parent artery-specific velocity profiles for the basilar artery (cases 1,6, and 9), internal carotid artery (cases 3, 7, and 8), middle cerebral artery (cases 4 and 5), and anterior cerebral artery (case 2). Median values for the maximum and minimum velocities were obtained from previous patient-specific $4 \mathrm{D}$ phase-contrast MR imaging velocity measure- 


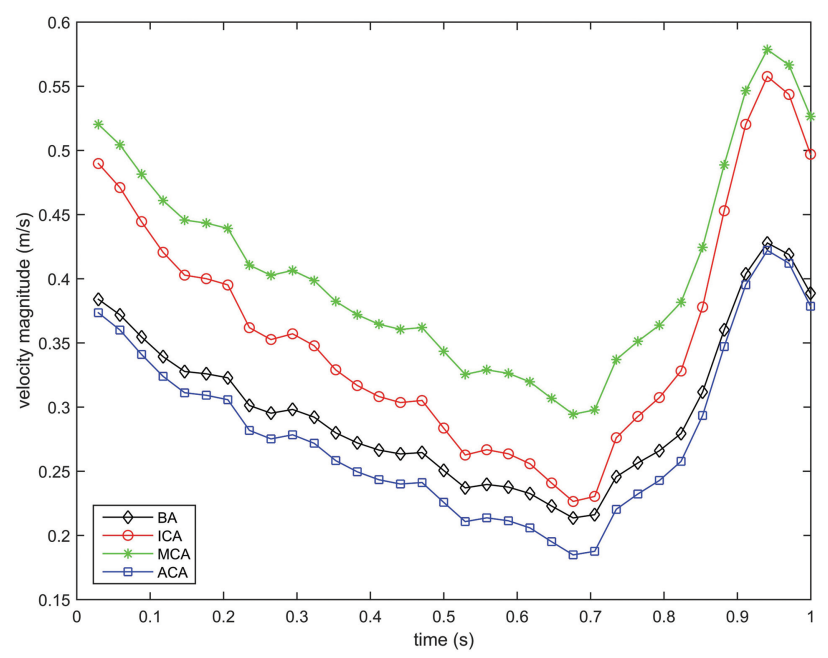

FIG 1. Velocity magnitude ( $\mathrm{m} / \mathrm{s}$ ) waveforms for the basilar artery (BA), internal carotid artery, middle cerebral artery, and the anterior cerebral artery (ACA).

ments. A middle cerebral artery velocity profile was used as a reference waveform to reconstruct velocity profiles consisting of 34 interpolated time-steps for each location (Fig 1).

Murray's law was used to determine the outflow ratio of the distal arteries. The third of 3 cardiac cycles was used for analysis. $^{28}$

\section{Qualitative Analysis}

Flow-velocity magnitude streamlines and WSS distributions were visualized by using Paraview 3.6.2 (Kitware; Los Alamos National Laboratory, Los Alamos, New Mexico). The visualizations were qualitatively assessed by 2 experienced neuroradiologists (C.B.L.M.M., R.v.d.B.) for hemodynamic characteristics in consensus. Flow complexity, flow stability, and the inflow concentration were assessed on the basis of visualization of the streamlines. ${ }^{29}$ The region and size of flow impingement were determined from the WSS distribution and streamlines.

\section{Quantitative Oscillatory Shear Index Measurements}

The mean and maximum oscillatory shear indices (OSIs) ${ }^{30}$ were calculated to assess the directional change of WSS during the cardiac cycle. Differences in OSI values before and after rupture were determined.

\section{RESULTS}

Streamlines at peak systole and heart cycle-averaged WSS distributions of all 18 vascular models are shown in Figs 2 and 3, respectively. All vascular models had a single inflow jet, single or multiple vortices, and at least 1 clear impingement zone before and after rupture. The location of maximum WSS and the region of flow impingement agreed for all cases. Hemodynamic differences were observed in 6 of the 9 aneurysms (Table 2 ). For the 6 patients with hemodynamic changes, the median time between imaging before and after rupture was 678 days (range, 14-1461 days). For the 3 patients without hemodynamic changes, this was 151 days (range, 34-183 days). Flow complexity changed from simple to complex in 3 cases. Flow stability changed from stable to unstable in 3 cases. The inflow concentration changed from diffuse to concentrated in 1 case and from concentrated to diffuse in another case. The region of flow impingement changed in 3 cases. These hemodynamic changes were associated with growth, aneurysm displacement, or newly formed lobulations. ${ }^{14}$ Changes in OSI after rupture ranged from -0.037 to +0.034 and from -0.030 to +0.12 for the spatially averaged and maximum values, respectively (Table 3).

\section{Qualitative Characterization: Case-by-Case Description}

For cases 1 and 6, aneurysmal geometry and hemodynamics did not change after rupture. In case 2, the aneurysm was displaced $8.9 \mathrm{~mm}$ after rupture, most likely due to mass effect from a large perianeurysmal hematoma (dimensions, $28 \times 22 \times 35 \mathrm{~mm}$ ). In this case, the region of flow impingement changed from the dome to the body. In the On-line Video, changes of WSS distributions before $(A)$ and after $(C)$ rupture with velocity magnitude streamlines before $(B)$ and after $(D)$ rupture of this case can be appreciated.

Case 3 showed a relative growth of $79 \%$ with 1 new lobulation after rupture. The flow profile changed from simple and stable to complex and unstable after rupture.

The aneurysm of case 4 was displaced $5.8 \mathrm{~mm}$ after rupture, most likely due to mass effect from a large perianeurysmal hematoma (dimensions, $46 \times 33 \times 38 \mathrm{~mm}$ ). However, there were no changes in hemodynamic characterizations.

In case 5 , a volumetric increase of $176 \%$ and a displacement of $1.2 \mathrm{~mm}$ after rupture were found. For the hemodynamic characterization, a diffuse inflow pattern before rupture changed to a concentrated one after rupture.

In case 7 , there was a relative growth of $832 \%$, with a newly observed lobulation in the imaging after rupture. The simple flow changed to a complex flow pattern after rupture. Also, an additional impingement zone was observed in the body of the aneurysm at the border of the lobulation after rupture.

In case 8 , there was a relative growth of $70 \%$ and a displacement of $2.5 \mathrm{~mm}$ after rupture. The flow pattern changed from stable to unstable. Before rupture, the inflow stream impacted the aneurysm at 2 regions: the aneurysm neck and body. After rupture, the neck of the aneurysm was the only region of impingement.

In case 9, a new lobulation was observed in the imaging after rupture. The flow changed from simple and stable to complex and unstable.

\section{DISCUSSION}

Most current rupture-risk identification studies compare models of ruptured aneurysms with unruptured models and ignore the possibility that the rupture itself may change aneurysm morphology and hemodynamics. However, our study shows that hemodynamic characteristics changed in 6 of the 9 aneurysms in the period between before and after imaging. Notably, we show changes in flow complexity, stability, and concentration, which have been associated with a risk of rupture. ${ }^{29}$

The altered hemodynamics are the consequence of a change in morphology in the period between image acquisitions. Because of the large imaging time interval in some cases, we cannot relate the 


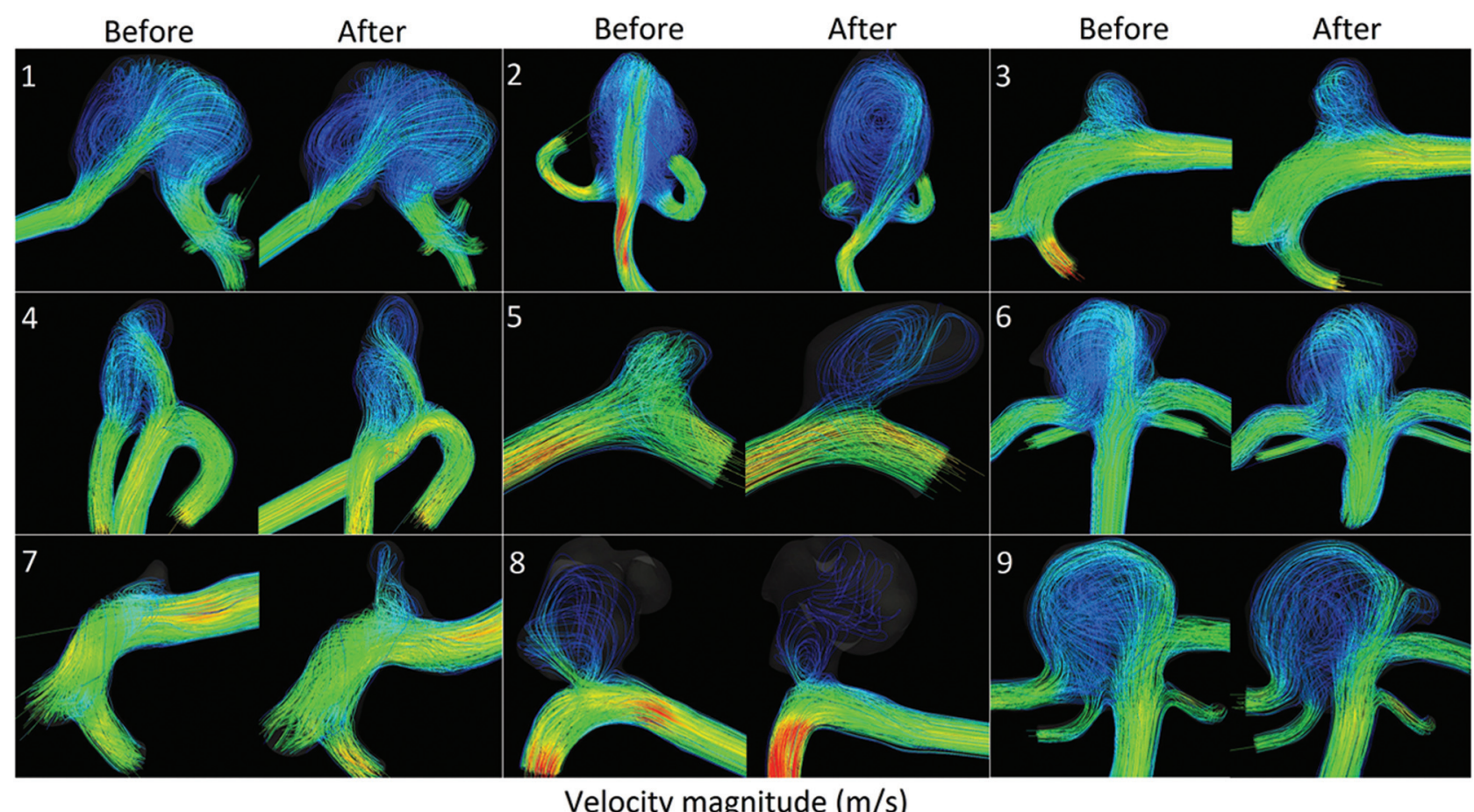

FIG 2. Flow-velocity streamlines $(\mathrm{m} / \mathrm{s})$ for all 9 aneurysms before and after rupture. For each aneurysm, the time-step with the peak velocitymagnitude of the inflow artery is shown.

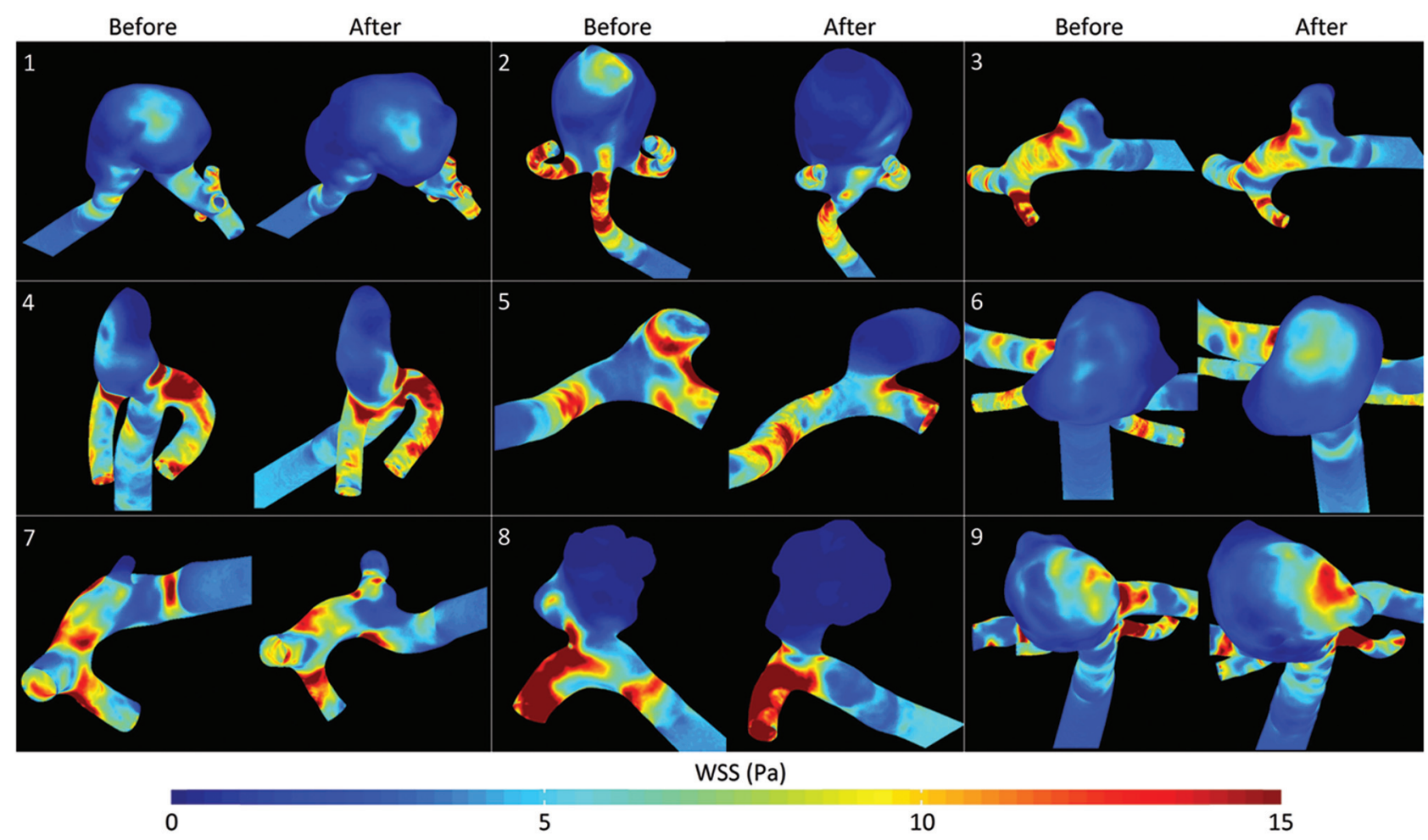

FIG 3. Heart cycle-averaged WSS (Pascal) distributions for all 9 aneurysms before and after rupture.

observed hemodynamic alterations to sudden, rupture-related morphologic changes alone. For instance, growth or lobulation formation may also have occurred more gradually during the whole time span. This scenario agrees with the median time be- tween before and after rupture imaging, which was much larger for the cases with hemodynamic changes than for the cases without. Furthermore, all of the 3 cases in which flow patterns changed from simple to complex showed a newly formed lobulation. How-

1930 Cornelissen Oct 2015 www.ajnr.org 
Table 2: Hemodynamic characteristics of the aneurysm models before and after rupture ${ }^{a}$

\begin{tabular}{|c|c|c|c|c|c|c|c|}
\hline \multirow[b]{2}{*}{$\mathrm{Pt}$} & \multirow[b]{2}{*}{ Location } & \multirow{2}{*}{$\begin{array}{c}\text { Flow } \\
\text { Complexity }\end{array}$} & \multirow{2}{*}{$\begin{array}{c}\text { Flow } \\
\text { Stability }\end{array}$} & \multirow{2}{*}{$\begin{array}{c}\text { Inflow } \\
\text { Concentration }\end{array}$} & \multicolumn{2}{|c|}{$\begin{array}{c}\text { Flow } \\
\text { Impingement }\end{array}$} & \multirow[b]{2}{*}{ Geometric Changes } \\
\hline & & & & & Region & Size & \\
\hline 1 & Basilar & $\mathrm{C}-\mathrm{C}$ & $U-U$ & $\mathrm{C}-\mathrm{C}$ & $D-D$ & S-S & None $^{\mathrm{b}}$ \\
\hline 2 & AcomA & $\mathrm{C}-\mathrm{C}$ & $U-U$ & $\mathrm{C}-\mathrm{C}$ & $D-B^{c}$ & S-S & Aneurysm displacement $(8.9 \mathrm{~mm})$ \\
\hline 3 & PcomA & $S-C^{c}$ & $S-U^{c}$ & $D-D$ & $\mathrm{~N}-\mathrm{N}$ & S-S & Growth (79\%); 1 new lobulation \\
\hline 4 & MCA & $\mathrm{C}-\mathrm{C}$ & $U-U$ & $\mathrm{C}-\mathrm{C}$ & ND-ND & S-S & Aneurysm displacement $(5.8 \mathrm{~mm})$ \\
\hline 5 & MCA & $\mathrm{C}-\mathrm{C}$ & S-S & $D-C^{c}$ & ND-ND & S-S & Growth (176\%) \\
\hline 6 & Basilar & $\mathrm{C}-\mathrm{C}$ & $U-U$ & $D-D$ & $D-D$ & $L-L$ & None ${ }^{b}$ \\
\hline 7 & PcomA & $S-C^{c}$ & $U-U$ & $C-D^{c}$ & $N-N B^{C}$ & S-S & Growth (832\%); 1 new lobulation \\
\hline 8 & Anterior choroidal & $\mathrm{C}-\mathrm{C}$ & $S-U^{c}$ & $\mathrm{C}-\mathrm{C}$ & $N B-N^{c}$ & S-S & Growth (70\%) \\
\hline 9 & Basilar & $\mathrm{S}-\mathrm{C}^{\mathrm{c}}$ & $S-U^{c}$ & $\mathrm{C}-\mathrm{C}$ & $B D-B D$ & L-L & 1 New lobulation \\
\hline
\end{tabular}

Note:-AcomA indicates anterior communicating artery; PcomA, posterior communicating artery.

a Flow complexity was characterized as simple (S) or complex (C); flow stability, as stable (S) or unstable (U); inflow concentration as diffuse (D) or concentrated (C); region of flow impingement, as dome (D), body (B), or neck (N); and size of the impingement zone, as small (S) or large (L).

${ }^{b}$ Aneurysm displacement of $\leq 0.6 \mathrm{~mm}$ or not related to mass effect from hematoma; aneurysm growth of $\leq 40 \%$.

${ }^{c}$ Changed after rupture.

Table 3: Mean and maximum oscillatory shear index for all 9 aneurysms before and after rupture

\begin{tabular}{|c|c|c|c|c|c|c|}
\hline \multirow[b]{2}{*}{ Pt } & \multicolumn{3}{|c|}{ Mean OSI } & \multicolumn{3}{|c|}{ Maximum OSI } \\
\hline & Before & After & $\Delta$ & Before & After & $\Delta$ \\
\hline 1 & 0.042 & 0.049 & +0.007 (17\%) & 0.487 & 0.484 & $-0.003(0.6 \%)$ \\
\hline 2 & 0.074 & 0.044 & $-0.029(40 \%)$ & 0.486 & 0.479 & $-0.007(1.4 \%)$ \\
\hline 3 & 0.025 & 0.049 & $+0.024(95 \%)$ & 0.423 & 0.482 & $+0.059(13.9 \%)$ \\
\hline 4 & 0.031 & 0.044 & $+0.013(42 \%)$ & 0.471 & 0.483 & $+0.012(2.5 \%)$ \\
\hline 5 & 0.007 & 0.040 & $+0.034(520 \%)$ & 0.350 & 0.469 & $+0.119(34.1 \%)$ \\
\hline 6 & 0.065 & 0.028 & $-0.037(57 \%)$ & 0.489 & 0.459 & $-0.030(6.1 \%)$ \\
\hline 7 & 0.061 & 0.031 & $-0.029(48 \%)$ & 0.471 & 0.476 & +0.005 (1.1\%) \\
\hline 8 & 0.021 & 0.018 & $-0.003(16 \%)$ & 0.475 & 0.452 & $-0.023(4.8 \%)$ \\
\hline 9 & 0.025 & 0.058 & $+0.034(137 \%)$ & 0.473 & 0.485 & $+0.011(2.4 \%)$ \\
\hline Mean & $0.039 \pm 0.023$ & $0.040 \pm 0.013$ & & $0.458 \pm 0.045$ & $0.474 \pm 0.012$ & \\
\hline
\end{tabular}

ever, morphologic changes may also occur suddenly, shortly before, during, or just after rupture. This change can be illustrated by the large growth of case 8 within 14 days and by the aneurysm displacement in both cases ( 2 and 4 ) with a hematoma with mass effect.

Therefore, hemodynamics of ruptured models may still be valuable for gradually changing aneurysms, where the value seems more limited for models with rupture-related morphologic changes. In most cases, it is difficult to discriminate between sudden and gradual changes in morphology, except for cases with large perianeurysmal hematomas. We recommend excluding these cases in studies that compare hemodynamic characteristics in ruptured and unruptured aneurysms because hematomas may strongly influence hemodynamics.

Only a few studies assessed hemodynamics both before and after rupture. In 4 aneurysms of the posterior communicating artery, Chien and Sayre ${ }^{31}$ determined that only 1 hemodynamic parameter (pulsatility index) was not affected by rupture. This agrees with our observations that most hemodynamic characteristics changed. Duan et $\mathrm{al}^{32}$ examined hemodynamic and morphologic parameters by using a case-control study of 6 aneurysms on the posterior communicating artery with hemodynamic data within 7 days before rupture. However, they only compared parameters with those of matched controls and did not investigate the influence of the rupture itself. This was also the case in the study of Liu et al, ${ }^{33}$ in which low WSS was associated with rupture in a case-control study of large internal carotid artery aneurysms, including 3 unruptured aneurysms just before rupture and 8 un- ruptured controls. A recent case report of an aneurysm with imaging before and just after rupture demonstrated changes in WSS values between $20 \%$ and $30 \%$, caused by a change in the aneurysm shape. $^{34}$

In a recent study by Bor et al, ${ }^{35}$ aneurysm growth was studied in a large population of patients with unruptured and untreated intracranial aneurysms. In their study, $>10 \%$ of the aneurysms grew during a mean follow-up time of $<3$ years. Growth is considered a marker for increased risk of rupture, and because growth may change hemodynamics, such a population allows improved characterization of hemodynamic rupture-risk factors.

A previous study by Xiang et $\mathrm{al}^{8}$ showed that high OSI values were associated with ruptured aneurysms. We indeed found high OSI values in all ruptured models. Most important, OSI was also high in all except 1 of the unruptured models. The exception was a case with $>3$ years between imaging, in which gradual progression from low-to-high shear stress oscillation might have been missed. These data therefore suggest that high OSI values may remain valuable for risk prediction.

\section{Limitations}

The current study has a number of limitations. Although this study is the largest comparing hemodynamic parameters within aneurysms before and after rupture, the number of patients was too small to perform statistical analysis. CFD is sensitive to the geometry and therefore image resolution, and neck size overestimation can affect the calculated hemodynamic parameters. ${ }^{19}$ Previous literature concluded that WSS calculations are strongly de- 
pendent on segmentation and resolution. ${ }^{36}$ Because of the large variation in resolution, the WSS estimations were not robust. Therefore, we used WSS distributions only for determining the impingement region, and no absolute WSS values were reported. Manual corrections for imaging artifacts were performed by a single neuroradiologist. These imaging artifacts were apparent in all cases; therefore, we do not expect large variations among different observers. Furthermore, common limitations for CFD studies, such as the generic pulsatile waveform, rigid wall assumption, limited accuracy of the algorithm, Newtonian fluid assumption, and CFD solution strategies may affect the accuracy of CFD results. $^{37-39}$

\section{CONCLUSIONS}

We have shown that morphologic changes before, during, or just after rupture may result in differences in hemodynamic characteristics between the ruptured and unruptured status of aneurysms. Hemodynamic differences were associated with aneurysm growth, lobulation formation, and displacement due to hematoma. Associations of hemodynamic characteristics with aneurysm rupture obtained from case-control studies comparing ruptured and unruptured aneurysms should, therefore, be interpreted with caution and not automatically used in risk models for aneurysm rupture.

Disclosures: Wouter Potters—RELATED: Other: Dutch Technology Foundation STW (Carisma 111629), * Comments: Salary was paid by a grant from a government granting agency (the Dutch Technology Foundation STW) for this submitted work. René van den Berg-UNRELATED: Consultancy: Codman/DePuy neurovascular consultancy in 2014. Birgitta Velthuis-UNRELATED: Payment for Lectures (including service on Speakers Bureaus): Philips Healthcare.* Ed VanBavel—UNRELATED: Grants/Grants Pending: European Union Marie Curie program. * Charles Majoie-RELATED: Grant: Nuts Ohra Foundation*; UNRELATED: Grants/Grants Pending: Dutch Heart Foundation. * Money paid to the institution.

\section{REFERENCES}

1. Brisman JL, Song JK, Newell DW. Cerebral aneurysms. N Engl J Med 2006;355:928-39

2. Vlak MH, Algra A, Brandenburg R, et al. Prevalence of unruptured intracranial aneurysms, with emphasis on sex, age, comorbidity, country, and time period: a systematic review and meta-analysis. Lancet Neurol 2011;10:626-36

3. Nieuwkamp DJ, Setz LE, Algra A, et al. Changes in case fatality of aneurysmal subarachnoid haemorrhage over time, according to age, sex, and region: a meta-analysis. Lancet Neurol 2009;8:635-42

4. Gabriel RA, Kim H, Sidney S, et al. Ten-year detection rate of brain arteriovenous malformations in a large, multiethnic, defined population. Stroke 2010;41:21-26

5. van Rooij WJ, Sprengers ME, de Gast AN, et al. 3D rotational angiography: the new gold standard in the detection of additional intracranial aneurysms. AJNR Am J Neuroradiol 2008;29:976-79

6. Greving JP, Wermer MJ, Brown RD, et al. Development of the PHASES score for prediction of risk of rupture of intracranial aneurysms: a pooled analysis of six prospective cohort studies. Lancet Neurol 2014;13:59-66

7. Cebral JR, Mut F, Weir J, et al. Quantitative characterization of the hemodynamic environment in ruptured and unruptured brain aneurysms. AJNR Am J Neuroradiol 2011;32:145-51

8. Xiang J, Natarajan SK, Tremmel M, et al. Hemodynamic-morphologic discriminants for intracranial aneurysm rupture. Stroke 2011;42:144-52

9. Backes D, Vergouwen MDI, Velthuis BK, et al. Difference in aneurysm characteristics between ruptured and unruptured aneurysms in patients with multiple intracranial aneurysms. Stroke 2014;45: 1299-303

10. Shojima M, Oshima M, Takagi $\mathrm{K}$, et al. Magnitude and role of wall shear stress on cerebral aneurysm: computational fluid dynamic study of $\mathbf{2 0}$ middle cerebral artery aneurysms. Stroke 2004;35: 2500-05

11. Meng H, Tutino VM, Xiang J, et al. High WSS or Low WSS? Complex interactions of hemodynamics with intracranial aneurysm initiation, growth, and rupture: toward a unifying hypothesis. AJNR Am J Neuroradiol 2014;35:1254-62

12. Xiang J, Tutino VM, Snyder KV, et al. CFD: computational fluid dynamics or confounding factor dissemination? The role of hemodynamics in intracranial aneurysm rupture risk assessment. AJNR Am J Neuroradiol 2014;35:1849-57

13. Rahman M, Ogilvy CS, Zipfel GJ, et al. Unruptured cerebral aneurysms do not shrink when they rupture: multicenter collaborative aneurysm study group. Neurosurgery 2011;68:155-60; discussion $160-61$

14. Schneiders JJ, Marquering HA, van den Berg R, et al. Rupture-associated changes of cerebral aneurysm geometry: high-resolution $3 \mathrm{D}$ imaging before and after rupture. AJNR Am J Neuroradiol 2014; 35:1358-62

15. Manniesing R, Velthuis BK, van Leeuwen MS, et al. Level set based cerebral vasculature segmentation and diameter quantification in CT angiography. Med Image Anal 2006;10:200-14

16. Cremers D, Rousson $M$, Deriche R. A review of statistical approaches to level set segmentation: integrating color, texture, motion and shape. Int J Comput Vis 2007;72:195-215

17. Luboz V, Wu X, Krissian $\mathrm{K}$, et al. A segmentation and reconstruction technique for 3D vascular structures. Med Image Comput Comput Assist Interv 2005;8(pt 1):43-50

18. Antiga L, Piccinelli M, Botti L, et al. An image-based modeling framework for patient-specific computational hemodynamics. Med Biol Eng Comput 2008;46:1097-112

19. Omodaka S, Inoue T, Funamoto K, et al. Influence of surface model extraction parameter on computational fluid dynamics modeling of cerebral aneurysms. J Biomech 2012;45:2355-61

20. Hoi Y, Woodward SH, Kim M, et al. Validation of CFD simulations of cerebral aneurysms with implication of geometric variations. J Biomech Eng 2006;128:844-51

21. Schneiders JJ, Marquering HA, Antiga L, et al. Intracranial aneurysm neck size overestimation with $3 \mathrm{D}$ rotational angiography: the impact on intra-aneurysmal hemodynamics simulated with computational fluid dynamics. AJNR Am J Neuroradiol 2013;34:121-28

22. Moench T, Neugebauer M, Preim B. Optimization of vascular surface models for computational fluid dynamics and rapid prototyping. In: Proceedings of the International Workshop on Digital Engineering, Magdeburg, Germany. November 21-22, 2011:16-23

23. Blender Foundation. Blender. Free Open 3D Creat Softw. 1995. http:// www.blender.org/. Accessed October 9, 2013

24. Yushkevich PA, Piven J, Hazlett HC, et al. User-guided 3D active contour segmentation of anatomical structures: significantly improved efficiency and reliability. Neuroimage 2006;31:1116-28

25. Taubin G. Curve and surface smoothing without shrinkage. In: Proceedings of the Fifth International Conference on Computer Vision, Boston, Massachusetts. June 22-23, 1995

26. Ortega J, Hartman J, Rodriguez J, et al. Post-treatment hemodynamics of a basilar aneurysm and bifurcation. Ann Biomed Eng 2008;36:1531-46

27. Caretto LS, Gosman AD, Patankar SV, et al. Two calculation procedures for steady, three-dimensional flows with recirculation. In: Cabannes H, Temam R, eds. Proceedings of the Third International Conference on Numerical Methods in Fluid Mechanics. New York, NY: Springer-Verlag; 1973:60-68

28. Cebral JR, Castro MA, Appanaboyina S, et al. Efficient pipeline for image-based patient-specific analysis of cerebral aneurysm 
hemodynamics: technique and sensitivity. IEEE Trans Med Imaging 2005;24:457-67

29. Cebral JR, Mut F, Weir J, et al. Association of hemodynamic characteristics and cerebral aneurysm rupture. AJNR Am J Neuroradiol 2011;32:264-70

30. Ku DN, Giddens DP, Zarins CK, et al. Pulsatile flow and atherosclerosis in the human carotid bifurcation. Positive correlation between plaque location and low oscillating shear stress. Arteriosclerosis 1985;5:293-302

31. Chien A, Sayre J. Morphologic and hemodynamic risk factors in ruptured aneurysms imaged before and after rupture. AJNR Am J Neuroradiol 2014;35:2130-35

32. Duan G, Lv N, Yin J, et al. Morphological and hemodynamic analysis of posterior communicating artery aneurysms prone to rupture: a matched case-control study. J Neurointerv Surg 2014 Nov 17. [Epub ahead of print]

33. Liu J, Fan J, Xiang J, et al. Hemodynamic characteristics of large unruptured internal carotid artery aneurysms prior to rupture: a case control study. J Neurointerv Surg $2015 \mathrm{Feb}$ 4. [Epub ahead of print]

34. Kono K, Tomura N, Yoshimura R, et al. Changes in wall shear stress magnitude after aneurysm rupture. Acta Neurochir (Wien) 2013; 155:1559-63

35. Bor AS, Tiel Groenestege AT, terBrugge KG, et al. Clinical, radiological, and flow-related risk factors for growth of untreated, unruptured intracranial aneurysms. Stroke 2015;46:42-48

36. Potters WV, van Ooij $\mathrm{P}$, Marquering $\mathrm{H}$, et al. Volumetric arterial wall shear stress calculation based on cine phase contrast MRI. $J$ Magn Reson Imaging 2015;41:505-16

37. Jansen IG, Schneiders JJ, Potters WV, et al. Generalized versus patient-specific inflow boundary conditions in computational fluid dynamics simulations of cerebral aneurysmal hemodynamics. AJNR Am J Neuroradiol 2014;35:1543-48

38. Dempere-Marco L, Oubel E, Castro M, et al. CFD analysis incorporating the influence of wall motion: application to intracranial aneurysms. Med Image Comput Comput Assist Interv 2006; 9:438-45

39. Valen-Sendstad K, Steinman DA. Mind the gap: impact of computational fluid dynamics solution strategy on prediction of intracranial aneurysm hemodynamics and rupture status indicators. AJNR Am J Neuroradiol 2014;35:536-43 